\title{
Assessing the progression of chronic periodontitis using subgingival pathogen levels: a 24-month prospective multicenter cohort study
}

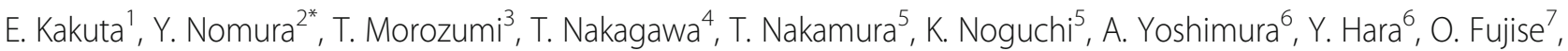

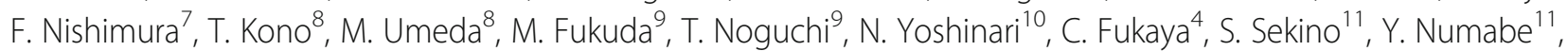
N. Sugano ${ }^{12}$, K. Ito ${ }^{12}$, H. Kobayashi ${ }^{13}$, Y. Izumi ${ }^{13}$, H. Takai ${ }^{14}$, Y. Ogata ${ }^{14}$, S. Takano ${ }^{15}$, M. Minabe ${ }^{15,16}$, A. Makino-Oi ${ }^{17}$, A. Saito ${ }^{17}$, Y. Abe ${ }^{18}$, S. Sato ${ }^{19}$, F. Suzuki ${ }^{20}$, K. Takahashi $^{21}$, T. Sugaya ${ }^{22}$, M. Kawanami ${ }^{22}$, N. Hanada ${ }^{2}$,

S. Takashiba ${ }^{23}$ and H. Yoshie ${ }^{3}$

\begin{abstract}
Background: The diagnosis of the progression of periodontitis presently depends on the use of clinical symptoms (such as attachment loss) and radiographic imaging. The aim of the multicenter study described here was to evaluate the diagnostic use of the bacterial content of subgingival plaque recovered from the deepest pockets in assessing disease progression in chronic periodontitis patients.

Methods: This study consisted of a 24-month investigation of a total of 163 patients with chronic periodontitis who received trimonthly follow-up care. Subgingival plaque from the deepest pockets was recovered and assessed for bacterial content of Porphyromonas gingivalis, Prevotella intermedia, and Aggregatibacter actinomycetemcomitans using the modified Invader PLUS assay. The corresponding serum IgG titers were measured using ELISA. Changes in clinical parameters were evaluated over the course of 24 months. The sensitivity, specificity, and prediction values were calculated and used to determine cutoff points for prediction of the progression of chronic periodontitis.
\end{abstract}

Results: Of the 124 individuals who completed the 24-month monitoring phase, 62 exhibited progression of periodontitis, whereas 62 demonstrated stable disease. The $P$. gingivalis counts of subgingival plaque from the deepest pockets was significantly associated with the progression of periodontitis $(p<0.001$, positive predictive value $=0.708)$.

Conclusions: The $P$. gingivalis counts of subgingival plaque from the deepest pockets may be associated with the progression of periodontitis.

Keywords: Progression of periodontitis, Periodontal stability, Porphyromonas gingivalis, Subgingival plaque

\section{Background}

Periodontitis is a chronic inflammation, which is leaded by periodontal bacteria and host immune response [1]. Periodontitis results in loss of connective tissue and bone support and is a major cause of tooth loss in adults [2]. The maintenance care following the initial active therapy phase plays an essential part in periodontal

\footnotetext{
* Correspondence: nomura-y@tsurumi-u.ac.jp

${ }^{2}$ Department of Translational Research, Tsurumi University School of Dental

Medicine, 2-1-3 Tsurumi, Tsurumi-ku, Yokohama 230-8501, Japan

Full list of author information is available at the end of the article
}

treatment to prevent disease progression based on the patient's individual needs [3].

The clinical diagnosis of chronic periodontal disease is based on visual and radiographic assessment of the periodontal tissues and on measurements of the space between the tooth and gum [2]. However, traditional clinical criteria are often insufficient for determining sites of active disease, for monitoring quantitatively the response to therapy or for measuring the degree of susceptibility to future disease progression [4]. Therefore, it is 
necessary to establish effective markers indicating periodontitis progression.

The role of salivary biomarkers and periodontal pathogens were determined during periodontal disease progression [5]. Salivary Porphyromonas gingivalis ratio (ratio: $P$. gingivalis counts/Total bacteria counts) may be potential indicators for the progression of periodontitis. Such a salivary test could be a useful diagnostic tool for predicting periodontal disease progression [6]. And also, our previous report suggested the $P$. gingivalis ratio would be an indicator for the progression of periodontitis [7]. Therefore, salivary periodontal pathogens were suggested as useful marker of periodontitis progression.

While, several previous studies determined the subgingival plaque pathogens were useful indicators of periodontal progression. Monitoring the proportions of $P$. gingivalis and Treponema denticola in subgingival plaque had the potential to help identify sites at significant risk for progression of periodontitis [8]. The levels of red complex bacteria ( $P$. gingivalis, Tannerella forsythia, and $T$. denticola) in subgingival plaque had a strong relationship with the clinical parameters [9]. Higher levels of $P$. gingivalis, Prevotella intermedia and Aggregatibacter actinomycetemcomitans in subgingival plaque appear to be related to an increased risk of periodontitis progression [10-12]. Similarly, A. actinomycetemcomitans, $P$. gingivalis, and $P$. intermedia may be useful as indicators of periodontitis activity [13]. In addition, presence of $A$. actinomycetemcomitans in subgingival plaque was a risk marker for periodontitis progression at the full mouth level [14, 15]. The increase in the number of $P$. gingivalis, $P$. intermedia in subgingival plaque of adolescents depends on the severity of gingivitis and appears to be a contributing factor for the shift from gingivitis to periodontitis [16, 17]. Thus, periodontal pathogens of subgingival plaque were valuable risk markers for periodontitis progression.

The bacterial tests or serum IgG tests were used to monitor periodontitis progress [5, 6, 18]. However, it remains unclear what periodontal bacteria from subgingival plaque are the most useful for estimation of periodontitis progression during regular follow-ups after initial therapy. In addition, previous reports did not test for possible correlations between the levels of periodontal bacteria in saliva and those in subgingival plaque from the deepest pockets.

The aims of this multicenter study were two-fold. The first goal was to evaluate subgingival bacteriological markers among patients treated for chronic periodontitis who exhibited or lacked subsequent disease progression during 24 months of regular follow-ups. The second goal was to define diagnostic values for indicating periodontitis progression and stability, including comparison between the levels of periodontal pathogens in subgingival plaque from the deepest pockets compared to those in saliva.

\section{Methods \\ Study design}

This study was performed as a clinical research project for diagnosis of periodontitis, as sponsored by the Japanese Society of Periodontology. Subjects were recruited in cooperation with 17 facilities (16 university hospitals and one clinic) in Japan, and were registered between February 2009 and February 2012. An additional file shows this in more detail [see Additional file 1]. The study enrolled a total of 163 follow-up patients, that is, individuals with chronic periodontitis who had completed active treatments such as initial therapy or periodontal surgery. Each diagnosis was based on the Guidelines of the American Academy of Periodontology [19]. All individuals were of age $\geq 30$ years, systemically healthy, possessed at least 20 teeth, and had not taken systemic antibiotics, anti-inflammatory drugs, or immunosuppressive drugs within 3 months prior to enrollment.

\section{Clinical assessment}

The follow-up patients were seen trimonthly over a 24month period. At each visit, the patients received treatment only to remove supragingival plaque and calculus, if detected. To identify disease progression, a full-mouth periodontal examination, except for the third molars, was performed every 6 months. The following clinical parameters were recorded: plaque index (PlI), bleeding on probing (BOP), probing pocket depth (PPD), and clinical attachment level (CAL). PII was recorded at 4 sites per tooth (mesial, buccal, distal, and lingual). PPD, CAL, and BOP were recorded at 6 sites per tooth (mesiobuccal, buccal, distobuccal, mesiolingual, lingual, and distolingual). Patients with at least one site of CAL loss of $\geq 3 \mathrm{~mm}$ at a given site over the 24-month study period were considered to be exhibiting periodontitis progression $[6,7,20,21]$. Intra- and inter-examiner calibration sessions were conducted at the beginning and middle of the study period.

\section{Sample collection}

At baseline, samples of subgingival plaque from the deepest pockets, saliva, and blood were collected from each patient. Samplings were carried out according to the following order; blood samples, saliva samples, and subgingival plaque samples. Whole saliva was collected from each subject by having the individual chew on a gum base, containing neither fragrance nor flavored ingredients, for $5 \mathrm{~min}$. The subgingival plaque was collected as follows: after removal of the supragingival plaque, samples of subgingival plaque from the deepest pockets (except for those at the third molars) were 
obtained by consecutive insertion (at a given site) of two sterile number-40 paper points (Zipperer Absorbent Paper Points, VDW GmbH, Munich, Germany) into the periodontal pocket for $10 \mathrm{~s}$ per point [22]. The plaque and saliva samples were sent immediately to a medical laboratory (BML Corporation, Tokyo, Japan) for bacterial analysis $[6,17,23]$. Blood sampling was performed using a commercial kit (DEMECAL Blood Test Set; Sunstar Inc, Osaka, Japan). In brief, device-treated serum was obtained from a $50-\mu \mathrm{L}$ sample of whole capillary blood collected from the middle fingertip. The samples of sera were sent immediately to a commercial laboratory (Leisure Inc., Tokyo, Japan) for immune analysis [7, 24-27].

\section{Quantification of periodontal bacteria in subgingival plaque from the deepest pockets and in saliva}

Quantitative analysis of the total bacterial count and periodontopathic bacterial counts, including $P$. gingivalis, $P$. intermedia, and $A$. actinomycetemcomitans, was performed using a modification of the Invader PLUS assay [7, 22, 28, 29]. Briefly, bacterial DNA was extracted from samples of the subgingival plaque from the deepest pockets by suspending each plaque sample in 1 $\mathrm{mL}$ of phosphate-buffered saline, $\mathrm{pH} 7.4$ and processing using the MagNA Pure LC Total Nucleic Acid Isolation Kit (Roche, Basel, Switzerland) according to the manufacturer's instructions. Similarly, bacterial DNA was extracted from the 100- $\mu \mathrm{l}$ whole saliva samples using the MagNA Pure LC Total Nucleic Acid Isolation Kit. The individual sequences of each bacterial species were obtained from a public database (National Center for Biotechnology Information, Bethesda, MD). Primers for each species were designed based on a region of the $16 \mathrm{~S}$ rRNA gene. A pair of universal primers and a universal probe were used to determine the total number of bacteria. Primary probes and Invader oligos were designed using the Invader technology creator (HOLOGIC, Madison, WI) and were based on sequences in the amplified regions [7, 28-30].

Template DNA was added to a $15-\mu \mathrm{L}$ reaction mixture containing primers for each species, $50 \mu \mathrm{M}$ dNTPs, $700 \mathrm{nM}$ primary probe, $70 \mathrm{nM}$ Invader oligo, $2.5 \mathrm{U}$ polymerase chain reaction (PCR) enzyme (EagleTaq DNA polymerase, Roche, Basel, Switzerland), and the Invader core reagent kit (Cleavase XI Invader core reagent kit, HOLOGIC, Madison, WI) containing a fluorescence resonance energy transfer (FRET) mix and an enzyme/ $\mathrm{MgCl}_{2}$ solution. The reaction mixture was preheated at $95{ }^{\circ} \mathrm{C}$ for $20 \mathrm{~min}$, and a 2 -step PCR reaction $\left(95^{\circ} \mathrm{C}\right.$ for $1 \mathrm{~s}$ and $63{ }^{\circ} \mathrm{C}$ for $1 \mathrm{~min}$ ) was performed for 35 cycles using an ABI PRISM 7900 thermocycler (Applied Biosystems, Foster City, CA). Fluorescence values of carboxyfluorescein (FAM; wavelength/bandwidth: excitation, $485 / 20 \mathrm{~nm}$; emission, $530 / 25 \mathrm{~nm}$ ) were measured at the end of the incubation/extension step at $63{ }^{\circ} \mathrm{C}$ for each cycle.

The limit of detection for this method was determined for each species with dilutions of bacterial DNA. Standard curves were constructed based on a crossing point determined by the fit point method.

Next, the proportions of the 3 pathogens within the total bacterial counts were determined [10,31]. Bacterial ratios (\%) and bacterial counts $\left(\log _{10}\right)$ for each species also were used in various comparison and diagnostic analyses.

\section{Measurement of IgG titers against periodontal bacteria}

Serum IgG antibody titers against periodontal pathogens were determined by Leisure Inc. using enzyme-linked immunosorbent assay (ELISA) [32]. The method and selection of bacterial antigens were based on previous reports [7, 24-27]. Briefly, sonicated preparations of $P$. gingivalis FDC381, $P$. intermedia ATCC25611, and $A$. actinomycetemcomitans ATCC29523 were used as representative bacterial antigens. Each antigen was diluted to $10 \mu \mathrm{g} / \mathrm{mL}$ with $0.1 \mathrm{M}$ carbonate buffer ( $\mathrm{pH}$ 9.6). An aliquot of this diluted solution $(100 \mu \mathrm{L})$ then was added to each well in a flat-bottomed microtiter plate (Greiner Co. Ltd., Frickenhausen, Germany) and the plate was stored overnight at $4{ }^{\circ} \mathrm{C}$. Each well with immobilized antigen was washed three times with PBS containing 0.05\% Tween-20 (PBST). Subsequently, a diluted serum sample (3100-fold dilution with PBST) was added to each well. After incubation at $37{ }^{\circ} \mathrm{C}$ for $2 \mathrm{~h}$, each well was washed three times with PBST and bound/free (B/F) separation was carried out. Next, a $100-\mu \mathrm{l}$ aliquot of 1:5000 diluted alkaline phosphatase-conjugated goat antihuman IgG antibody (Jackson Immuno Research Laboratories, Inc., Baltimore, MD) was added to each well. After incubation at $37{ }^{\circ} \mathrm{C}$ for $2 \mathrm{~h}$, each well was washed three times with PBST and B/F separation was carried out. Thereafter, $50 \mu \mathrm{l}$ of $p$-nitrophenyl phosphate (Wako Pure Chemical Industries, Ltd., Osaka, Japan) adjusted to $1 \mathrm{mg} / \mathrm{ml}$ with $10 \%$ diethanolamine buffer ( $\mathrm{pH}$ 9.8) was added to each well as substrate. The plate then was incubated at room temperature for $10-20 \mathrm{~min}$. The enzymatic reaction was terminated by adding $50 \mu \mathrm{l}$ of $3 \mathrm{M} \mathrm{NaOH}$, and optical density (measurement at $405 \mathrm{~nm}$; reference at $490 \mathrm{~nm}$ ) was measured in a Micro ELISA Auto Reader (Bio-Rad Laboratories, Hercules, CA).

The standard titration curves were formulated using serial dilutions of pooled control serum collected from participants without periodontitis. The absorbance of each sample after reaction was defined as an ELISA unit (EU), such that $100 \mathrm{EU}$ corresponded to 1:3200 dilution of the control sample. The antibody titers were expressed as standardized values calculated as follows: (EU for study serum samples-EU for control samples)/2 standard 
deviations (SD), using SDs as determined based on ELISA of the control samples [25].

\section{Statistical analysis}

Descriptive analysis of the collected data was performed; the results are presented as medians. The Mann Whitney U test was used to compare clinical parameters, periodontal bacterial amounts, and IgG titers with or without progression of chronic periodontitis (CP). Only correlation with sex was performed using the Fisher's exact test. To determine an optimal test for distinguishing follow-up patients with periodontal progression or stability, we constructed receiver operating characteristic (ROC) curves and set the cutoff point based on the minimum difference between sensitivity and specificity $[6,25]$. On the basis of the calculated cutoff point, we then made appropriate cross-tabulations. Diagnostic values were calculated by the Fisher's exact test to identify statistically significant differences. To check the correlation of periodontal bacteria with disease progression, logistic regression analysis was carried out to eliminate possible confounding variables. This study was originally designed to evaluate the diagnostic salivary levels of $P$. gingivalis in patients with or without the progression of CP. Calculation of the sample size was described in our previous report [7]. For the present study, post-hoc power analysis calculation using the cross table for patients with or without progression of $\mathrm{CP}$ against positive or negative for $P$. gingivalis suggested that groups would have to consist of at least 41 subjects each. A similar analysis revealed that groups of at least 26 subjects each would be required to detect differences in $P$. gingivalis counts $\left(\log _{10}\right)$ in subgingival plaque from the deepest pockets. All analyses were performed by independent statisticians (Y.N., E.K., and N.H.) using IBM SPSS Statistics ver. 19.0 software (IBM Japan, Tokyo, Japan) and S-plus (ver. 6.0, NTT DATA, Tokyo, Japan).

\section{Results}

Of the 163 follow-up patients enrolled in the study, 32 dropped out during the study period due to a variety of causes (including occurrence of the Great East Japan Earthquake), and 7 others withdrew because of the use during the study of antimicrobial agents for the treatment of acute periodontal abscesses. In the end, 124 subjects successfully completed the study protocol. Of the 124 patients who completed the study protocol, 62 follow-up patients $(50 \%)$ presented with disease progression, whereas the same number of patients $(50 \%)$ demonstrated stable disease.

Demographic characteristics and biological variables of the study participants at baseline are shown in Table 1 .

Table 1 Clinical parameters and bacterial levels at baseline in the patients with stable CP and progressive CP

\begin{tabular}{|c|c|c|c|}
\hline & Stable CP patients $(n=62)$ & Progressive CP patients $(n=62)$ & $p$-value \\
\hline Gender (male/female) & $23 / 39$ & $26 / 36$ & 0.199 \\
\hline Age (year) & $60.0(56.0-66.0)$ & $61(52.5-68.0)$ & 0.193 \\
\hline Smoking (non-smoker/smoker) & $59 / 3$ & $61 / 1$ & 0.619 \\
\hline PII & $0.12(0.06-0.30)$ & $0.17(0.07-0.45)$ & 0.291 \\
\hline BOP (\% positive) & $3.62(1.53-11.28)$ & $6.54(2.48-10.45)$ & $0.022^{*}$ \\
\hline $\operatorname{PPD}(\mathrm{mm})$ & $2.15(1.80-2.53)$ & $2.26(1.76-2.57)$ & 0.885 \\
\hline The deepest PPD (mm) & $4.0(4.00-6.00)$ & $5.0(4.25-7.75)$ & $<0.001^{*}$ \\
\hline $\mathrm{CAL}(\mathrm{mm})$ & $2.8(2.34-3.33)$ & $2.87(2.78-3.62)$ & 0.710 \\
\hline The deepest CAL (mm) & $7.00(5.25-8.00)$ & $7.5(6.25-9.00)$ & $0.042^{*}$ \\
\hline P. gingivalis counts $\left(\log _{10}\right)$ Subgingival plaque & $1.00(1.00-3.17)$ & $1.64(1.00-4.29)$ & $<0.001^{*}$ \\
\hline P. gingivalis counts $\left(\log _{10}\right)$ Saliva & $2.59(1.00-3.66)$ & $3.34(2.67-4.08)$ & $0.002^{*}$ \\
\hline P. gingivalis lgG titers (EU) & $1.28(0.32-4.26)$ & $1.63(0.95-6.34)$ & 0.503 \\
\hline P. intermedia counts $\left(\log _{10}\right)$ Subgingival plaque & $1.00(1.00-1.63)$ & $1.00(1.00-2.72)$ & $0.049^{*}$ \\
\hline P. intermedia counts $\left(\log _{10}\right)$ Saliva & $1.87(1.00-3.65)$ & $3.02(1.41-4.05)$ & $0.038^{*}$ \\
\hline P. intermedia lgG titers (EU) & $-0.39(0.47-0.13)$ & $-0.27(0.45-0.21)$ & 0.173 \\
\hline A. actinomycetemcomitans counts $\left(\log _{10}\right)$ Subgingival plaque & $1.00(1.00-1.00)$ & $1.00(1.00-1.00)$ & 0.164 \\
\hline A. actinomycetemcomitans counts $\left(\log _{10}\right)$ Saliva & $1.00(1.00-1.00)$ & $1.00(1.00-1.50)$ & 0.065 \\
\hline A. actinomycetemcomitans IgG titers (EU) & $-0.26(0.41-0.03)$ & $-0.30(0.40-0.10)$ & 0.278 \\
\hline
\end{tabular}

Value represent median value (25-75 percentile). $C P$ chronic periodontitis, $P / l$ plaque index, $B O P$ bleeding on probing, $P P D$ probing pocket depth, $C A L$ clinical attachment level, EU enzyme-linked immunosorbent assay unit

The deepest PPD and the deepest CAL indicate the value of the sites which is the deepest PPD and the deepest CAL in each subject, respectively. $p$ values for gender were calculated using the Fisher's exact test, and all others were Mann Whitneys' $\mathrm{U}$ tests. The ${ }^{*}$ mark represent statistical significance between stable $\mathrm{CP}$ patients and progressive CP patients $(p<0.05)$ 

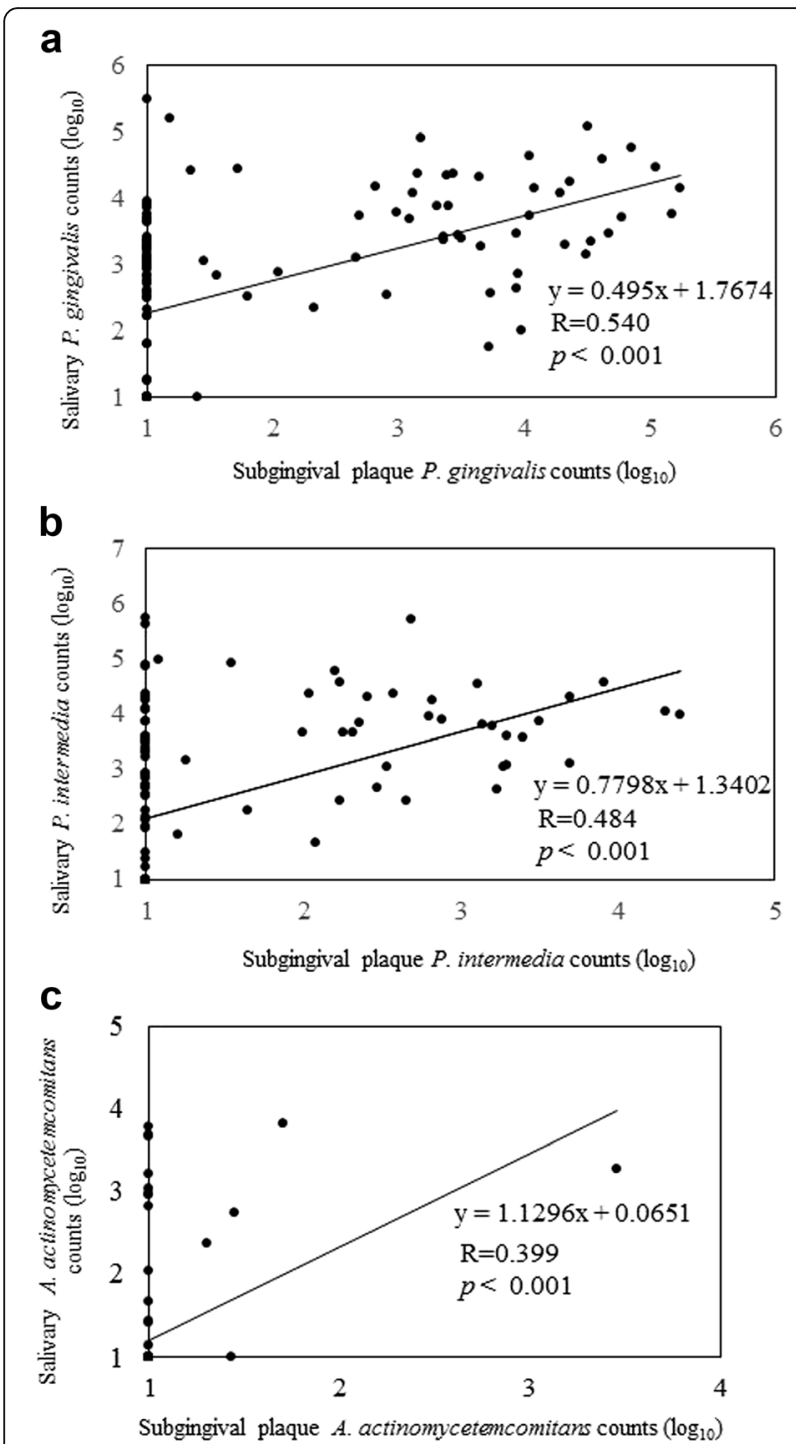

Fig. 1 Scatter plots of periodontal pathogen counts from salivary and subgingival plaque from the deepest pockets for individual subjects. Correlations of periodontal pathogen counts in saliva and subgingival plaque from the deepest pockets were statistically significant. The correlation coefficient of bacterial counts in saliva and subgingival plaque from the deepest pockets was the highest for $P$. gingivalis (panel a) when compared with other pathogens (panel b, c). Note, for each of the three bacterial species, that bacterial counts were below the limit of detection in some subgingival plaque samples; this observation was most notable for A. actinomycetemcomitans, which could not be detected in $>95 \%$ of samples (panel c).

BOP, the deepest PPD, and the deepest CAL were significantly higher in subjects who exhibited progressive CP compared to patients with stable CP. For the pathogens, $P$. gingivalis counts in subgingival plaque from the deepest pockets, $P$. intermedia counts in subgingival plaque from the deepest pockets, $P$. gingivalis counts in saliva, and $P$. intermedia counts in saliva were significantly higher in subjects who exhibited progressive CP compared to patients with stable CP.

Figure 1 shows the scatter plots of periodontal pathogen counts in saliva compared to those in subgingival plaque from the deepest pockets. Correlation coefficients of bacterial counts from saliva and subgingival plaque from the deepest pockets were $0.540(p<0.001)$ for $P$. gingivalis, $0.484(p<0.001)$ for $P$. intermedia, and $0.399(p<0.001)$ for $A$. actinomycetemcomitans. However, in some samples of subgingival plaque from the deepest pockets, bacterial counts were below the limit of detection. Notably, A. actinomycetemcomitans was not detected in $95.7 \%$ of samples of subgingival plaque from the deepest pockets.

To determine an optimal test for distinguishing followup patients with periodontal progression from those with stable CP, we constructed receiver operating characteristic curves and set the cutoff points on the basis of the minimum differences between sensitivity and specificity $[6,25]$. Furthermore, on the basis of the cutoff point, we made appropriate cross-tabulations.

Table 2 shows the cutoff points, sensitivity, specificity, positive predictive value, negative predictive value, likelihood, and area under the curve for the periodontal bacterial counts and IgG titers. Among the 3 periodontal pathogens, only P. gingivalis yielded counts that demonstrated significant correlation with $\mathrm{CP}$ status (cutoff point $=1.370(p<0.001)$, sensitivity $=0.548$, specificity $=$ 0.774 , positive predictive value $=0.708$, negative predictive value $=0.632$, likelihood ratio $=2.429$, AUC $=0.666$ ) .

The area under the curve of the $P$. gingivalis counts was the largest amongst all markers evaluated. The specificity of $P$. gingivalis counts in subgingival plaque from the deepest pockets was higher than that for salivary P. gingivalis counts (0.323) [7].

To check the importance of periodontal bacteria in the progression of periodontal disease, logistic regression analysis was carried out to eliminate possible confounding variables. As shown in Table 3, the crude odds ratio of the deepest CAL in each subject, and $P$. gingivalis count were statistically significant. According to the multivariate adjusted odds ratio, only $P$. gingivalis counts exhibited a significant $(p=0.034)$ correlation with CP status.

\section{Discussion}

In this study, we evaluated potential markers for periodontitis progression during a 24-month study interval. The results suggested associations between periodontitis progression and bacterial levels in subgingival plaque recovered from the deepest pockets. We previously used PCR to examine the presence of each of six species of periodontopathic bacteria in whole saliva and subgingival plaque [33]. A statistical relationship was found between the presence of $P$. gingivalis, $P$. intermedia, Prevotella nigrescens, and $T$. denticola in saliva and in periodontal pocket samples; in 
Table 2 Diagnostic values of subgingival plaque bacterial levels and serum lgG titers for periodontal bacteria

\begin{tabular}{|c|c|c|c|c|c|c|c|c|}
\hline & Cutoff & $p$-value & Sensitivity & Specificity & $\begin{array}{l}\text { Positive predictive } \\
\text { value }\end{array}$ & $\begin{array}{l}\text { Negative predictive } \\
\text { value }\end{array}$ & Likelihood ratio & AUC \\
\hline P. gingivalis counts $\left(\log _{10}\right)$ & 1.370 & $<0.001$ & 0.548 & 0.774 & 0.708 & 0.632 & 2.429 & 0.666 \\
\hline P. intermedia counts $\left(\log _{10}\right)$ & 1.040 & 0.077 & 0.371 & 0.774 & 0.622 & 0.552 & 1.643 & 0.568 \\
\hline $\begin{array}{l}\text { A. actinomycetemcomitans } \\
\left.\text { counts ( } \log _{10}\right)\end{array}$ & 1.151 & 0.171 & 0.065 & 0.984 & 0.800 & 0.513 & 4.000 & 0.478 \\
\hline P. gingivalis lgG titer $^{\mathrm{a}}$ & 1.355 & $>0.999$ & 0.516 & 0.500 & 0.508 & 0.508 & 1.032 & 0.444 \\
\hline P. intermedia lgG titer ${ }^{a}$ & -0.345 & 0.281 & 0.565 & 0.548 & 0.556 & 0.557 & 1.250 & 0.535 \\
\hline $\begin{array}{l}\text { A. actinomycetemcomitans } \\
\text { lgG titer }{ }^{2}\end{array}$ & -0.275 & 0.590 & 0.452 & 0.484 & 0.467 & 0.469 & 0.875 & 0.571 \\
\hline
\end{tabular}

Fisher's exact test was used for statistical analysis $(p<0.05)$. The $P$. gingivalis counts was statistically significant

The area under the curve of the $P$. gingivalis counts was the largest amongst all markers. Subgingival plaque $P$. gingivalis counts specificity was higher than salivary P. gingivalis counts (0.323) [7]

$E U$ enzyme-linked immunosorbent assay unit, $A U C$ area under the curve

a. J Periodontal Res. 2016 Dec;51(6):768-778

the event of disagreement, the organisms were more frequently present in saliva than in periodontal pockets $(p<0.001)$. Meanwhile, A. actinomycetemcomitans and T. forsythia were not reliably detected by sampling of either saliva or periodontal pockets [31].

Our results indicated that The $P$. gingivalis counts of subgingival plaque from the deepest pockets might be used as indicators of the progression of periodontitis. The results of the present study, along with those of our previous study [7], suggest the value of $P$. gingivalis in assessing the progression of CP. Specificity of $P$. gingivalis counts in subgingival plaque from the deepest pockets was higher than that in saliva. The absolute pathogen burden is clearly more informative than merely a dichotomization between carriers and noncarriers [34]. P. gingivalis, alone or in combination with T. forsythia and T. denticola, has been reported to exhibit the deepest mean pocket depths [9]. The thresholds predictive of progressive periodontitis correspond to $0.01 \%$ for A. actinomycetemcomitans, $0.1 \%$ for $P$. gingivalis, and $2.5 \%$ for $P$. intermedia [13]. In an 18-month-long study, the presence of $P$. gingivalis and $P$. intermedia in subgingival plaque at proportions exceeding $2 \%$ was a significant predictor of periodontitis progression in older adults [35]. These results support the appropriateness using $P$. gingivalis as a marker for the progression of $\mathrm{CP}$, and are consistent with the results obtained in the present study. In the work described here, we evaluated the diagnostic value for periodontal disease of the levels of bacteria in subgingival plaque from the deepest pockets (Table 2), in extension of our previous report [7] showing the diagnostic value for periodontal disease of salivary bacterial levels. Specificity, positive predictive value, likelihood ratio, and AUC of $P$. gingivalis counts for the progression of periodontal disease

Table 3 Multiple logistic regression analysis for progression of chronic periodontitis

\begin{tabular}{|c|c|c|c|c|}
\hline & Crude OR (95\% CI) & $p$-value & Multivariate adjusted OR (95\% Cl) & $p$-value \\
\hline Gender (male/female) & $0.62(0.30-1.29)$ & 0.200 & $1.06(0.43-2.65)$ & 0.893 \\
\hline Age (years) & $1.02(0.98-1.07)$ & 0.271 & $1.03(0.97-1.08)$ & 0.324 \\
\hline PII & $2.15(0.37-12.48)$ & 0.393 & $0.88(0.10-8.10)$ & 0.909 \\
\hline BOP (\% positive) & $1.04(1.00-1.08)$ & 0.077 & $1.03(0.98-1.08)$ & 0.285 \\
\hline $\mathrm{CAL}(\mathrm{mm})$ & $1.14(0.69-1.87)$ & 0.603 & $0.72(0.33-1.58)$ & 0.415 \\
\hline The deepest CAL (mm) & $1.25(1.01-1.54)$ & $0.039^{*}$ & $1.35(0.99-1.85)$ & 0.059 \\
\hline P. gingivalis counts $\left(\log _{10}\right)$ & $4.163(1.913-9.06)$ & $<0.001^{*}$ & $1.56(1.03-2.34)$ & $0.034^{*}$ \\
\hline P. gingivalislgG titers (EU) & $1.01(0.98-1.05)$ & 0.422 & $1.01(0.99-1.03)$ & 0.276 \\
\hline P. intermedia counts $\left(\log _{10}\right)$ & $2.022(0.92-4.443)$ & 0.080 & $0.99(0.52-1.76)$ & 0.973 \\
\hline P. intermedia lgG titers (EU) & $1.45(0.46-4.60)$ & 0.531 & $2.04(0.50-8.22)$ & 0.318 \\
\hline A. actinomycetemcomitans counts $\left(\log _{10}\right)$ & $4.207(0.457-38.757)$ & 0.205 & $44.70(0.05-36,574)$ & 0.267 \\
\hline A. actinomycetemcomitans lgG titers (EU) & $0.78(0.33-1.85)$ & 0.581 & $0.52(0.15-1.76)$ & 0.291 \\
\hline
\end{tabular}

The crude odds ratio of the deepest CAL in each subject, P. gingivalis count, and the combination of P. gingivalis counts and P. gingivalis IgG titers were statistically significant. According to the multivariate adjusted odds ratio, only P. gingivalis counts was statistically significant $(p=0.034)$

OR odds ratio, $95 \% \mathrm{Cl}$ confidence interval with $95 \%$ significance level, $P$ Il plaque index, BOP bleeding on probing, CAL clinical attachment level;Counts, each bacteria counts in subgingival plaque, $E U$ enzyme-linked immunosorbent assay unit ${ }^{*} p<0.05$ 
in subgingival plaque were higher than the respective parameters for bacterial counts in saliva. However, we note that bacterial counts could not be determined in numerous samples of subgingival plaque from the deepest pockets, with detection failing for $P$. gingivalis, $P$. intermedia, and A. actinomycetemcomitans in 59.7, 70.2 , and $95.7 \%$ of samples, respectively.

Subgingival plaque samples from the deepest pockets did not yield detectable levels of periodontal bacteria in several individuals enrolled in this study; however, the salivary samples were able to detect periodontal bacteria in these cases. An inherent limitation of sampling subgingival plaque from the deepest pockets is that these specimens only reflect the condition of sampling site. Notably, however, $P$. gingivalis counts in subgingival plaque from the deepest pockets yielded greater sensitivity and specificity for periodontal progression than did $P$. gingivalis counts in saliva. In this context, it is worth noting that sampling of subgingival plaque from the deepest pockets is more difficult than sampling of saliva. Clinicians using these tests should consider these merits and demerits when considering the use of sampling for periodontal bacteria in subgingival plaque from the deepest pockets. Further investigations will be needed to assess the utility of salivary bacteria levels as markers of periodontal condition.

\section{Conclusions}

The $P$. gingivalis counts of subgingival plaque from the deepest pockets may be associated with the progression of periodontitis.

\section{Additional file}

Additional file 1: List of the ethics committees and approval numbers. (XLSX $12 \mathrm{~kb})$

\section{Acknowledgements}

Not applicable.

\section{Funding}

This study was supported by a grant of the clinical research project for the diagnosis of periodontitis from the Japanese Society of Periodontology.

\section{Availability of data and materials}

The clinical data and personal details will not be made available in order to protect the participants' identity.

\section{Authors' contributions}

We confirmed that all authors have read and approved the final version of the manuscript, and agreed with this submission to your journal. Also, we are confident that all authors meet conditions the ICMJE authorship criteria. EK performed statistical analysis and prepared the manuscript. TM performed follow-up care of patients, data collection and prepared the manuscript. TNakag planed study design and managed recruitment of subjects. TNakam, KN, AY, YH, OF, TK, MF, TNog, NY, CF, SSek, YNuma, NS, Kl, HK, Yl, HT, YO, STakan, MM, AM-O, YA, SSa, FS, and TS, MK, NH performed follow-up care of patients and data collection. FN, MU, AS and KT advised regarding bacterial and serum aspects, and divided the preparation of the manuscript. YNomu and $\mathrm{NH}$ performed statistical analysis. STakash managed database administration. HY planed study design and gave scientific advices on the study.

\section{Competing interests}

The authors declare that they have no competing interests.

Consent for publication

Not applicable.

Ethics approval and consent to participate

Subjects were recruited in cooperation with 17 facilities (16 university hospitals and one clinic) in Japan, and were registered between February 2009 and February 2012. Informed written consent was obtained from each subject, and the protocol was approved by the Institutional Review Board of each participating institution. An additional file shows this in more detail [see Additional file 1].

\section{Author details}

${ }^{1}$ Department of Oral Microbiology, Tsurumi University School of Dental Medicine, 2-1-3 Tsurumi, Tsurumi-ku, Yokohama, Japan. Department of Translational Research, Tsurumi University School of Dental Medicine, 2-1-3 Tsurumi, Tsurumi-ku, Yokohama 230-8501, Japan. ${ }^{3}$ Division of Periodontology, Department of Oral Biological Science, Niigata University Graduate School of Medical and Dental Sciences, 2-5274 Gakkocho-dori, Chuo-ku, Niigata, Japan. ${ }^{4}$ Department of Dentistry and Oral Surgery, School of Medicine, Keio University, 35 Shinano-machi, Shinjuku-ku, Tokyo, Japan. ${ }^{5}$ Department of Periodontology, Kagoshima University Graduate School of Medical and Dental Sciences, 8-35-1 Sakuragaoka, Kagoshima, Japan. ${ }^{6}$ Department of Periodontology, Unit of Translational Medicine, Nagasaki University Graduate School of Biomedical Sciences, 1-12-4 Sakamoto, Nagasaki, Japan. ${ }^{7}$ Section of Periodontology, Division of Oral Rehabilitation, Faculty of Dental Science, Kyushu University, 3-1-1 Maidashi, Higashi-ku, Fukuoka, Japan. ${ }^{8}$ Department of Periodontology, Osaka Dental University, 8-1 Kuzuhahanazonocho, Hirakata, Japan. ${ }^{9}$ Department of Periodontology, School of Dentistry, Aichi Gakuin University, 2-11 Suemori-doori,Chikusa-ku, Nagoya, Japan. ${ }^{10}$ Department of Periodontology, School of Dentistry, Matsumoto Dental University, 1780 Hirokagobara, Shiojiri, Nagano, Japan.

${ }^{11}$ Department of Periodontology, School of Life Dentistry at Tokyo, The Nippon Dental University, 1-9-20 Fujimi, Chiyoda-ku, Tokyo, Japan.

${ }^{12}$ Department of Periodontology, Nihon University School of Dentistry, 1-8-13 Kanda-Surugadai, Chiyoda-ku, Tokyo, Japan. ${ }^{13}$ Department of Periodontology, Graduate School of Medical and Dental Sciences, Tokyo Medical and Dental University, 1-5-45 Yushima, Bunkyo-ku, Tokyo, Japan. ${ }^{14}$ Department of Periodontology, Nihon University School of Dentistry at Matsudo, 2-870-1 Sakae-cho-nishi, Matsudo-shi, Chiba, Japan. ${ }^{15}$ Bunkyo-Dori Dental Clinic, 2-4-1 Anagawa, Inage-ku, Chiba, JAPAN. ${ }^{16}$ Division of Periodontology, Department of Oral function and Restoration, School of Dentistry, Kanagawa Dental University, 82 Inaokacho, Yokosuka, Kanagawa, Japan. ${ }^{17}$ Department of Periodontology, Tokyo Dental College, 2-9-18 Misakicho, Chiyoda-ku, Tokyo, Japan. ${ }^{18}$ Comprehensive Dental Care, The Nippon Dental University Niigata Hospital, 1-8 Hamaura-cho, Chuo-ku, Niigata, Japan. ${ }^{19}$ Department of Periodontology, School of life Dentistry at Niigata, The Nippon Dental University, 1-8 Hamaura-cho, Chuo-ku, Niigata, Japan. ${ }^{20}$ Division of Dental Anesthesiology, Department of Oral Surgery, School of Dentistry, Ohu University, 31-1 Misumido, Tomita, Koriyama, Fukushima, Japan. ${ }^{21}$ Division of Periodontics, Department of Conservative Dentistry, School of Dentistry, Ohu University, 31-1 Misumido, Tomita, Koriyama, Fukushima, Japan. ${ }^{22}$ Division of Periodontology and Endodontology, Department of Oral Health Science, Hokkaido University Graduate School of Dental Medicine, Kita 13, Nishi 7, Kita-ku, Sapporo, Japan. ${ }^{23}$ Department of Pathophysiology-Periodontal Science, Okayama University Graduate School of Medicine, Dentistry and Pharmaceutical Sciences, 2-5-1 Shikata-cho, Okayama, Japan.

Received: 24 September 2016 Accepted: 6 January 2017

Published online: 16 January 2017

\section{References}

1. Salvi GE, Lang NP. Host response modulation in the management of periodontal diseases. J Clin Periodontol. 2005;32:108-29.

2. Pihlstrom BL, Michalowicz BS, Johnson NW. Periodontal diseases. Lancet. 2005;366:1809-20

3. Jansson L, Lagervall M. Periodontitis progession in patients subjected to supportive maintenance care. Swed Dent J. 2008;32:105-14. 
4. Giannobile W, Beikler T, Kinney JS, Ramseier CA, Morelli T, Wong DT. Saliva as a diagnostic tool for periodontal disease: current state and future directions. Periodontol 2000. 2009;50:52-64.

5. Kinney JS, Morelli T, Braun T, et al. Saliva/pathogen biomarker signatures and periodontal disease progression. J Dent Res. 2011;90:752-8.

6. Nomura Y, Shimada Y, Hanada N, et al. Salivary biomarkers for predicting the progression of chronic periodontitis. Arch Oral Biol. 2012;57:413-20.

7. Morozumi T, Nakagawa T, Nomura Y, et al. Salivary pathogen and serum antibody to assess the progression of chronic periodontitis: a 24-mo prospective multicenter cohort study. J Periodontal Res. 2016 Jan 20. doi: 10.1111/jre.12353. [Epub ahead of print]

8. Byrne SJ, Dashper SG, Darby IB, Adams GG, Hoffmann B, Reynolds EC. Progression of chronic periodontitis can be predicted by the levels of Porphyromonas gingivalis and Treponema denticola in subgingival plaque. Oral Microbiol Immunol. 2009;24:469-77.

9. Socransky SS, Haffajee AD, Cugini MA, et al. Microbial complexes in subgingival plaque. J Clin Periodontol. 1998;25:134-44.

10. Haffajee AD, Socransky SS, Smith C, Dibart S. Relation of baseline microbia parameters to future periodontal attachment loss. J Clin Periodontol. 1991 18:744-50.

11. Haffajee AD, Socransky SS. Microbial etiological agents of destructive periodontal diseases. Periodontol. 1994;5:78-111. Review.

12. Wang $X, L i ~ L$, Yang $M$, Geng $Y$, Chen $H, X u Y$, Sun $Y$. Prevalence and distribution of Aggregatibacter actinomycetemcomitans and its cdtB gene in subgingival plaque of Chinese periodontitis patients. BMC Oral Health. 2014;14:37. doi:10.1186/1472-6831-14-37.

13. Bragd L, Dahlén $G$, Wikström M, Slots J. The capability of Actinobacillus actinomycetemcomitans, Bacteroides gingivalis and Bacteroides intermedius to indicate progressive periodontitis; a retrospective study. J Clin Periodontol. 1987;14:95-9.

14. Van der Velden U, Abbas F, Armand S, Loos BG, Timmerman MF, et al. Java project on periodontal diseases. The natural development of periodontitis: risk factors, risk predictors and risk determinants. J Clin Periodontol. 2006;33:540-8.

15. Timmerman MF, Van der Weijden GA, Abbas F, et al. Untreated periodontal disease in Indonesian adolescents. Longitudinal clinical data and prospective clinical and microbiological risk assessment. J Clin Periodontol. 2000;27:932-42.

16. Papapanou PN, Baelum V, Luan WM, et al. Subgingival microbiota in adult Chinese: prevalence and relation to periodontal disease progression. J Periodontol. 1997;68:651-66.

17. Yang NY, Zhang Q, Li JL, Yang SH, Shi Q. Progression of periodontal inflammation in adolescents is associated with increased number of Porphyromonas gingivalis, Prevotella intermedia, Tannerella forsythensis, and Fusobacterium nucleatum. Int J Paediatr Dent. 2014;24:226-33.

18. Sugi N, Naruishi K, Kudo C, et al. Prognosis of periodontitis recurrence after intensive periodontal treatment using examination of serum $\lg \mathrm{G}$ antibody titer against periodontal bacteria. J Clin Lab Anal. 2011;25:25-32.

19. Krebs KA, Clem 3rd DS. American Academy of Periodontology, Guidelines for the management of patients with periodontal diseases. J Periodontol. 2006;77:1607-11.

20. Beck JD. Methods of assessing risk for periodontitis and developing multifactorial models. J Periodontol. 1994;65:468-78.

21. Ogawa H, Yoshihara A, Hirotomi T, Ando Y, Miyazaki H. Risk factors for periodontal disease progression among elderly people. J Clin Periodontol. 2002:29:592-7.

22. Morozumi T, Kubota T, Abe D, Shimizu T, Komatsu Y, Yoshie H. Effects of irrigation with an antiseptic and oral administration of azithromycin on bacteremia caused by scaling and root planning. J Periodontol. 2010;81: $1555-63$.

23. Morozumi T, Kubota T, Abe D, Shimizu T, Nohno K, Yoshie H. Microbiological effect of essential oils in combination with subgingival ultrasonic instrumentation and mouth rinsing in chronic periodontitis patients. Int J Dent. 2013;2013:146479.

24. Takeuchi K, Furuta M, Takeshita T, et al. Serum antibody to Porphyromonas gingivalis and periodontitis progression: the Hisayama Study. J Clin Periodontol. 2015:42:719-25.

25. Kudo C, Naruishi K, Maeda H, et al. Assessment of the plasma/serum lgG test to screen for periodontitis. J Dent Res. 2012;91:1190-5.

26. Kudo C, Shin WS, Minabe M, et al. Analysis of the relationship between periodontal disease and atherosclerosis within a local clinical system: a cross-sectional observational pilot study. Odontology. 2015;103:314-21.
27. Furuta M, Shimazaki Y, Tanaka S, et al. Gender-specific associations of serum antibody to Porphyromonas gingivalis and inflammatory markers. Biomed Res Int. 2015;2015:897971.

28. Tadokoro K, Yamaguchi T, Kawamura K, et al. Rapid quantification of periodontitis-related bacteria using a novelmodification of Invader PLUS technologies. Microbiol Res. 2010;165:43-9.

29. Tada A, Takeuchi H, Shimizu H, et al. Quantification of periodontopathic bacteria in saliva using the invader assay. Jpn J Infect Dis. 2012:65:415-23.

30. Lyons SR, Griffen AL, Leys EJ. Quantitative real-time PCR for Porphyromonas gingivalis and total bacteria. J Clin Microbiol. 2000;38:2362-5.

31. Tomita S, Komiya-Ito A, Imamura K, et al. Prevalence of Aggregatibacter actinomycetemcomitans, Porphyromonas gingivalis and Tannerella forsythia in Japanese patients with generalized chronic and aggressive periodontitis. Microb Pathog. 2013;61-62:11-5.

32. Murayama $Y$, Nagai $A$, Okamura $K$, et al. Serum immunoglobulin $G$ antibody to periodontal bacteria. Adv Dent Res. 1988;2:339-45.

33. Umeda M, Contreras A, Chen C, Bakker I, Slots J. The utility of whole saliva to detect the oral presence of periodontopathic bacteria. J Periodontol. 1998;69:828-33.

34. Papapanou PN, Neiderud AM, Papadimitriou A, Sandros J, Dahlén G "Checkerboard" assessments of periodontal microbiota and serum antibody responses: a case-control study. J Periodontol. 2000;71:885-97.

35. Brown LF, Beck JD, Rozier RG. Incidence of attachment loss in communitydwelling older adults. J Periodontol. 1994;65:316-23.

\section{Submit your next manuscript to BioMed Central and we will help you at every step:}

- We accept pre-submission inquiries

- Our selector tool helps you to find the most relevant journal

- We provide round the clock customer support

- Convenient online submission

- Thorough peer review

- Inclusion in PubMed and all major indexing services

- Maximum visibility for your research

Submit your manuscript at www.biomedcentral.com/submit
) Biomed Central 